Supplementary Information

\title{
Reversible Ionic Polarization in Metal Halide Perovskites
}

\author{
Zhibo Yan ${ }^{1 *}$, Wenjing Zhai ${ }^{1}$, Feng Wang ${ }^{2 *}$, Qian $\mathrm{Li}^{1}$, Lin Lin ${ }^{1}$, Chunxiong Bao ${ }^{2}$, \\ Chunfeng Zhang ${ }^{1}$, Feng $\mathrm{Gao}^{2 *}$, and J. -M. Liu ${ }^{1,3}$
}

\section{A detailed explanation for the TPC based on bulk ionic polarization mechanism.}

There is a symmetrical energy band structure in the $\mathrm{Au} /$ perovskite/Au device before the poling, as illustrated in Figure $\mathrm{S} 1(\mathrm{a})$. After the poling with $V_{p}=100 \mathrm{~V}$, however, the ionic polarization makes the energy band bending and produces negative/positive screen charges at the left/right electrodes respectively, as shown in Figure $\mathrm{S} 1(\mathrm{~b})$. As the device is under the light illumination and with $V_{b}=0$ [Figure $\mathrm{S} 1(\mathrm{c})$ ], the local dipoles would separate the photocarriers, which in turn acts as the screen charges to screen the build-in field induced by the polarization. As a result, the original negative/positive screening charges at the electrodes starts to discharge, forming a negative transient photocurrent. Then as most of the original screen charges has been discharged, the transient photocurrent is reduced and only a small negative $I_{s c}$ is left because the incompletely screen of polarization can still separate the photocarriers. Therefore, a valley-like overshoot photocurrent relaxation displays during the light

\footnotetext{
${ }^{1}$ Laboratory of Solid State Microstructures and Innovation Center of Advanced Microstructures, Nanjing University, Nanjing 210093 (P. R. China). E-amil: zbyan@nju.edu.cn

2 Department of Physics, Chemistry, and Biology (IFM), Linköping University, Linköping SE-581 83, Sweden. Email: feng.wang@liu.se, feng.gao@liu.se

${ }^{3}$ Institute for Advanced Materials and Laboratory of Quantum Engineering and Materials, South China Normal University, Guangzhou 510006, China
} 
pulse. As the light pulse is switched off, the photocarriers is quickly decayed and no enough to screen the polarization, therefore it requires to drawn back the charges from external circuit to compensate the absence of screen charges before the photocurrent decays to zero. Thus, a peak-like overshoot relaxation appears after the light pulse is switched off.

As $V_{b}<0$, the energy difference between the two electrodes is raised, which would help more photocarriers to be extracted out through electrodes, as shown in Figure S1(d), and hence explains the down-shift of the overshoot photocurrent curve in Figure 3(d). In contrast, as $V_{b}>0$, the energy band slopes down to the right side but the energy band bending near the electrodes remains due to the presence of ionic polarization, as illustrated in Figure S1(e). When the device is under the light illumination, the newly generated photocarriers act as the screen charge and the original screen charges are firstly discharged to form a negative transient photocurrent, then as the discharging is exhausted a positive photocurrent will be finally recovered due to the positive $V_{b}$, which explains the overshoot TPC as $V_{b} \geq 1 \mathrm{~V}$ in Figure 3(d).

The Poling tuned overshooting relaxation. Figure S2 (a) and (b) show the TPC after the poling with $V_{p}=100 \mathrm{~V}$ and $-100 \mathrm{~V}$ respectively. The poling with $V_{p}=100 \mathrm{~V}$ and -100 $\mathrm{V}$ causes to inversed overshoot relaxation behavior, and leads to negative and positive $I_{s c}$ respectively. It indicates that the TPC behavior is reversible and controllable by the poling voltage. Besides, the peak in the inset in Figure S2 (b) is delayed with the raise of $V_{b}$, which indicates that the peak is not caused by the flying of photocarriers from the left to the right electrodes but is the intrinsic photoelectric response. 

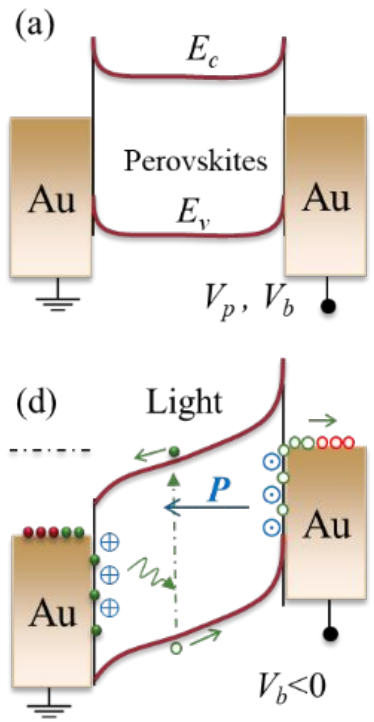
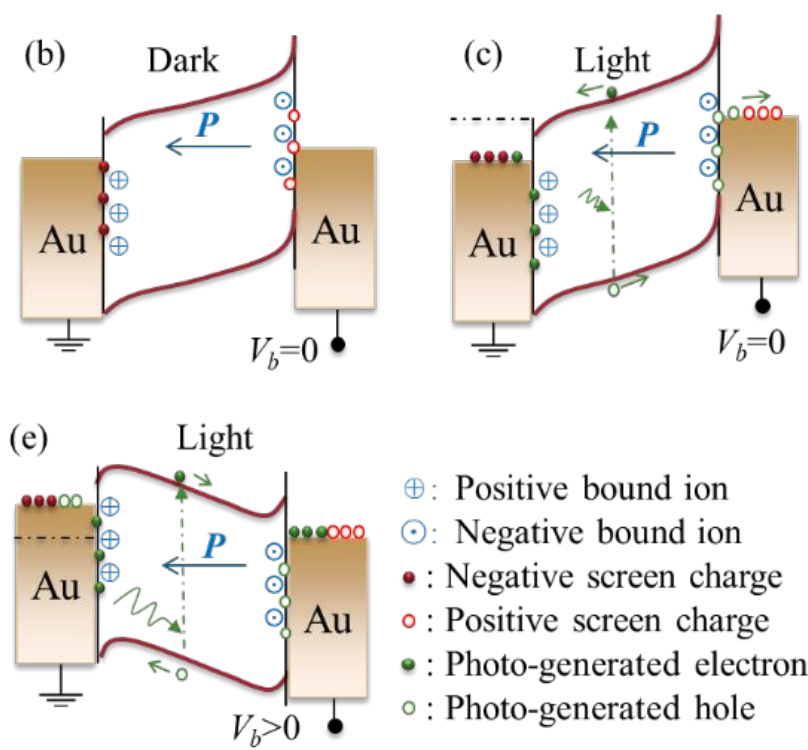

$\oplus$ : Positive bound ion

$\odot$ : Negative bound ion

- : Negative screen charge

$\circ$ : Positive screen charge

- : Photo-generated electron

$\circ$ : Photo-generated hole

Figure S1. The schematic energy band of Au/perovskite/Au before and after the poling with $V_{p}=100$ V. (a) Before the poling. (b) After the poling and with $V_{b}=0$ in the dark. The polarization induces the energy band bending and the negative/positive screening charges near left/right electrodes respectively. (c) After the poling and with $V_{b}=0$ in the light. The original negative/positive screen charges flow out through left/right electrodes respectively and photo-generated photocarriers acts as the new screen charges. (d) After the poling and with $V_{b}<0$ in the light. The energy difference between the two electrodes is raised and the more photocarriers are extracted out through electrodes. (e) After the poling and with $V_{b}>0$ in the light. The original negative/positive screen charges firstly flow out and then the photogenerated holes/electrons are followed through the left/right electrode respectively.
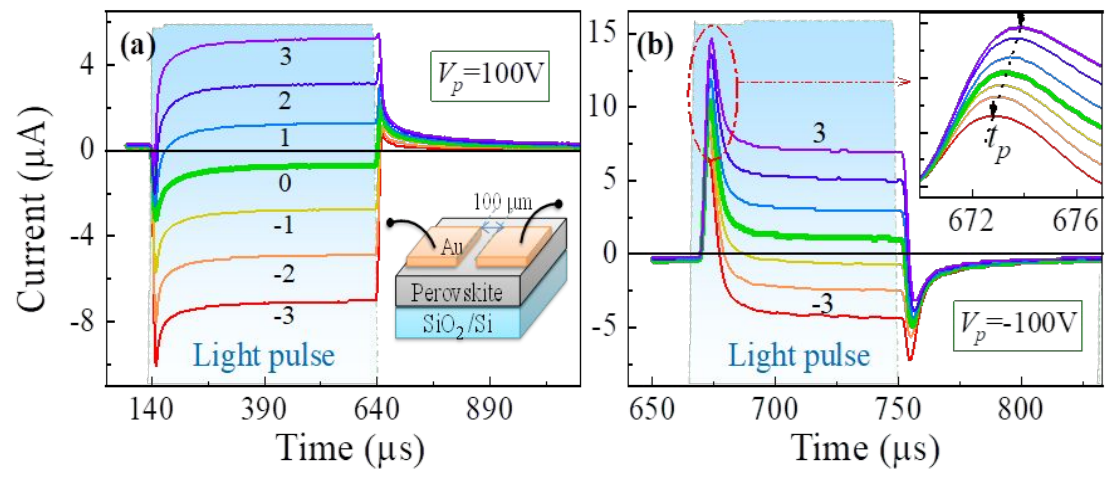

Figure S2. The transient photocurrent (TPC) at $200 \mathrm{~K}$ after the poling from $300 \mathrm{~K}$ with (a) $V_{p}=100$ $\mathrm{V}$ and (b) $V_{p}=-100 \mathrm{~V}$, with the bias varied from $-3 \mathrm{~V}$ to $3 \mathrm{~V}$, respectively. The inset in (a): the configuration of device. The inset in (b): enlarged view of TPC $\left(V_{p}=-100 \mathrm{~V}\right)$. 

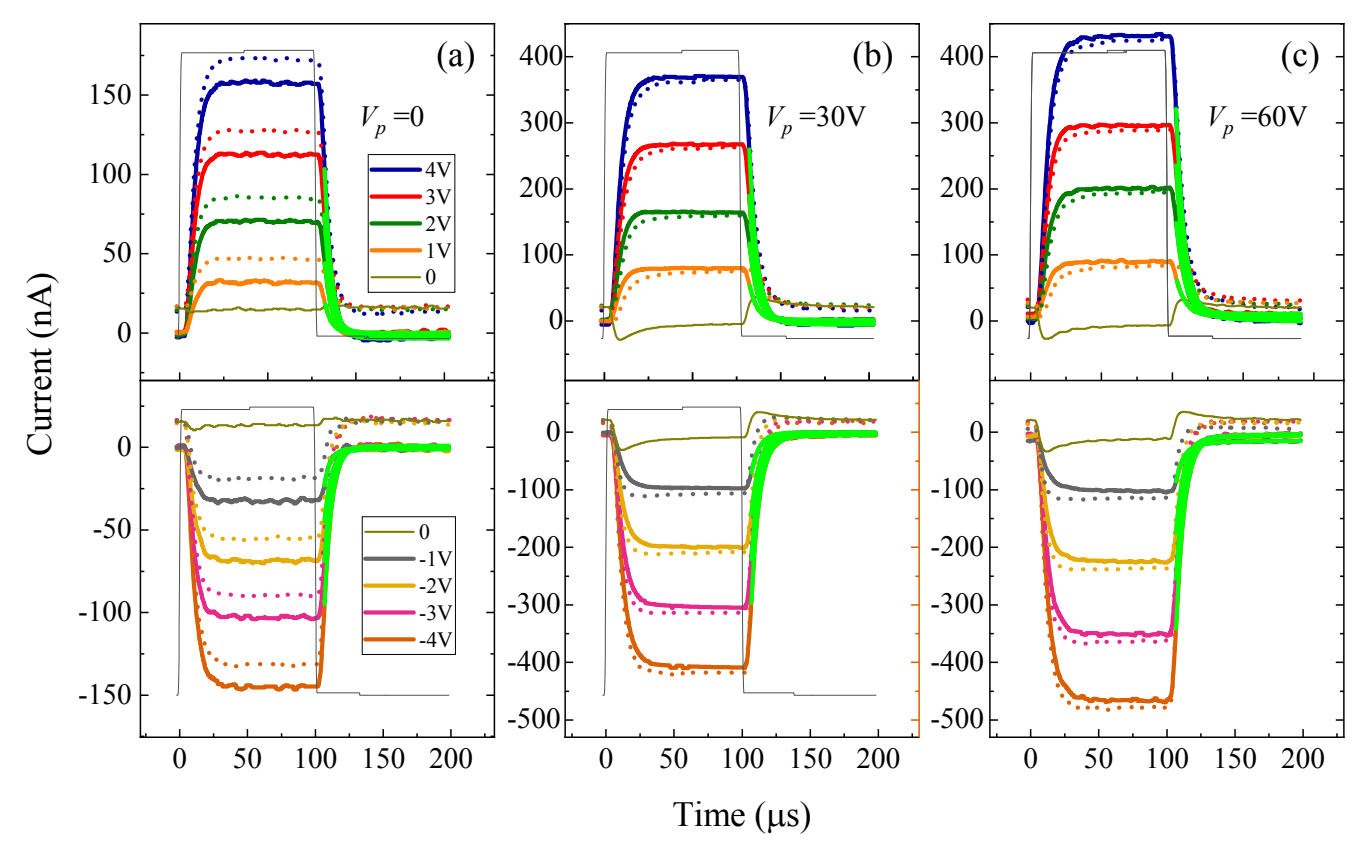

Figure S3 The TPC at $200 \mathrm{~K}$ after the poling of $V_{p}=0,30 \mathrm{~V}, 60 \mathrm{~V}$ respectively. The dot lines are measured TPCs under the various bias, and the solid lines are the TPCs minus a reference data (TPC under bias $=0$ ). The green curves are the fitting data of a single exponential decay model. 